\title{
How to Keep a Secret
}

\section{Chloe Mayne}

if you should stumble upon

a secret in your travels,

i warn you, take haste -

with a ruler determine the length

of its sharp, needly teeth

for some secrets will gnaw away at you

and leave nothing but the bones.

with a pair of scales determine its weight,

for the burden of a secret is sometimes

best shared with another

who is strong enough to carry it.

finally, with a sharp poke determine its temper

for some secrets cannot be tamed

and should be packed away in boxes, kept

far from the light. 